\title{
Eingeschobene Gedanken zum „Bildungsroman“. Zur Genrefrage
}

\section{1}

Strau $\beta$ endete beim Parzival und einer infernalisch inszenierten Rückkehr zur Grossen Mutter. Das verband ihn (für unseren Zusammenhang glücklich weiterführend) mit dem späteren Bildungs- und Entwicklungsroman als einem (insonderheit seit Wilhelm Dilthey) besonderen „deutschen“ Romanformat, hier bereits mehrfach erwähnt. Im allgemeinen literaturgeschichtlichen Bewusstsein gilt (eine Zuschreibung, im Zusammenhang mit Karl Lachmanns Übersetzung und Kommentierung des Nibelungenliedes entstanden) der Parzival als die mittelalterliche Vorstufe des Bildungsromans. In Eschenbachs Versepos geht es ja bereits um die Ausbildung des anfänglich noch ganz „reinen Toren" zu einem vollwertigen Mitglied der höfischen Feudalgesellschaft; wobei der "Minne“ ein ganz hoher Stellenwert zugewiesen wird, was wiederum der Frau, als der neu eingesetzten Erzieherin des höfischen Mannes, eine enorme Bedeutung einräumte. Adolf Muschg in Der rote Ritter hat daraus vor Jahren einen Prosaroman gemacht, sprachlich wie aus schwerer, dunkler Seide gewebt, ein kulturgeschichtlich hoch facettiertes Bild des Mittelalters zugleich. Zu diesem Vorhaben haben den Schweizer Autor eben jene vorweggenommenen Elemente des Bildungsromans in Wolfram von Eschenbachs Versepos verlockt (wobei der Zürcher sich ein Stück weit zu seiner „Vorlage“ ganz so verhielt, wie vor ihm Novalis zu Goethes Meister als dem Muster aller Bildungsromane). Eben um die letztgenannte frühromantische Adaption des Goethe'schen Musterstückes wird es im Folgenden gehen, getreu unserem Erkenntnisinteresse, wie es durch die „Blutsbrüderschaft“" Kafkas mit Heinrich von Kleist vorgegeben wurde. Dass der Begriff der Bildung zu dem Schlüsselwort in Literatur sowie pädagogischer Praxis des 18. Jahrhunderts geworden war, lässt sich anhand des Wörterbuchs der Brüder Grimm belegen. Die „Bildung“ eines Menschen konnte sowohl die

(C) Der/die Autor(en) 2021

B. Neumann, Umrisse einer Dritten Kultur im interdisziplinären Zusammenspiel zwischen Literatur und Naturwissenschaft, ELECTRISCHER PROMETHEUS. Umrisse einer Dritten Kultur im interdisziplinären Zusammenspiel zwischen Literatur und Naturwissenschaft, https://doi.org/10.1007/978-3-662-63204-8_11 
Bedeutung von Bildnis (,imago“) besitzen, wie auch die körperliche Gestalt bezeichnen (,forma“). Vor allem ging es freilich um dessen innere Verfeinerung im Sublimationsvorgang von Erziehung und Ausbildung, und das eben auch seit der mittelalterlichen Etablierung des Ritterideals im Versepos. Zur Verbesserung der natürlichen Anlagen des Menschen berief man sich später dann in der Renaissance, in Neuentdeckung der antiken Vorbilder, u. a. auf den Begriff der „cultura animi“, wie ihn Cicero in seinen Tusculae disputationes bereits im Jahr 45. V. Christi entwickelt hatte. Der Begriff der „Kultur“, der hier noch seine ursprüngliche Bedeutung von Fruchtbarmachung des Bodens mitführte, wurde auf die Veredelung und Vervollkommnung der menschlichen Anlagen transferiert: „Wie ein Acker, auch wenn er fruchtbar ist, ohne Pflege keine Frucht tragen kann, so auch die Seele nicht ohne Belehrung. Jede ist ohne das andere wirkungslos. Pflege der Seele aber ist Philosophie: sie zieht das Laster mit der Wirkung aus." " Aus diesem römischen Vorbild entstand dann im Mittelalter ein kulturgeschichtlich ganz neues Ideal, die Verbesserung des Menschen qua Erziehung, mithin durch Kultureinfluss; wurde danach in der Renaissance wiederentdeckt und, seit Gutenbergs Erfindung der Buchdruckkunst, durch Lesen in Eigenregie betrieben als ein immerwährender Lernprozess auf ein ethisches Ziel hin - Schillers Ästhetische Erziehung des Menschengeschlechts als Endziel. Der entscheidende Unterschied lag freilich darin, dass solche Kultivierung der menschlichen „Natur“ jetzt durch die Kulturleistung des Lesens betrieben werden sollte, mithin eine nachmittelalterliche Gesellschaft voraussetzend, in der es nicht nur gedruckte Bücher gab, sondern in der tendenziell alle des Lesens mächtig waren. So gelangte man zum Aufklärungsideal der individuellen Mündigkeit durch begründbares eigenes Urteil, zur Kant'schen Republik, in der, noch einmal, selbst Teufel noch zur Humanität sollten erzogen werden können. Die neu etablierte Literaturgesellschaft beinhaltete nun auch, dass sich der Roman, Hegels „,bürgerliche Epopoe“, als die endgültig dominierende Kunstform durchsetzte. Im Bildungsroman zum Medium der „Veredelung des Menschen“ erhoben, geriet die literarische Gattung des Romans insgesamt zu dem Medium einer Verständigung über neu erwachten Individuationswünsche und Selbstbestimmungshoffnungen des nunmehr auch ökonomisch aufsteigenden Bürgertums, logischerweise in jenen Staaten zuerst, in denen der moderne Kapitalismus sich etablierte (in

\footnotetext{
${ }^{1}$ Marcus Tullius Cicero, Gespräche in Tusculum, Lateinisch-Deutsch, hrsg. v. Olav Gigon, München 1970, S. 125.
} 
England und Holland, aber auch, unter modifizierten Bedingungen, in Deutschland samt der Habsburgischen Monarchie mit Einschluss Böhmens).

Hier sind weder Platz noch Notwendigkeit zugegen, eine differenzierte Geschichte des Bildungsromans (oder wirklich eingehende Interpretation des Goethe'schen Wilhelm Meister) zu geben. Was zuvörderst interessiert, ist dessen romantische Adaption durch Novalis, dessen Beförderung im kunsttheoretischen Denken der Brüder Schlegel und anderer führender romantische Köpfe zum Gegenstück zu Goethes Text. Immer gilt, was Robert Musil, auch ein guter Kafka-Bekannte, einmal lakonisch bemerkt hat: „Wenn Bildungsroman gesagt wird, schwebt Meister mit." ${ }^{2}$ Das erscheint so wahr wie lakonisch; ist auch Resultat der erst von Wilhelm Dilthey, dann durch Georg von Lukács in seiner vormarxistischen Periode, und später auch von Friedrich Gundolf im „George-Kreis“ betriebenen „Formierung“ des deutschen Geistes im letzten Deutschen Kaiserreich, im „Wilhelminismus“.3 Solche Bestrebungen waren dann im preussischen Berlin, und bei Dilthey gewiss, mit dem bereits schaudernd erahnten, weil als umstürzlerisch verdächtigtem neuen Weltbild der EinsteinÄra konnotiert. Diltheys wilhelminisches Erfolgsbuch Das Erlebnis und die Dichtung verrät den ideologischen Sachverhalt, der sich in seinem Fall bis auf das Barock und das Newton/Leibniz'sche Zeitalter zurückbezog. Also insgesamt auf Zeiten, in denen noch die Vorstellung ,prägend gewesen (war), dass der Mensch in eine von Gott gewollte Ordnung hineingeboren wird und dort eine ihm vorbestimmte soziale Position einnimmt". ${ }^{4}$ Dieser kaiserliche Untertan sollte sich um den immer noch sonnengleich im Mittelpunkt stehenden Monarchen auf fester sozialer Bahn so herum bewegen, wie die Planeten um das Zentralgestirn Sonne, und insonderheit Heinrich Manns Der Untertan wusste warum. Diese Vorstellung veränderte sich freilich mit dem Beginn des 20. Jahrhunderts, als sie revolutionär ausser Kraft gesetzt wurde von Hofmannsthal, Broch und auch Musil, mit Einschluss des Autors Kafka, wenn auch nicht unbedingt mit dem des Beamten. Das alles geschah nicht vorbildlos. Sondern im Bereich der (Roman) Kunst eben bereits in der frühen Jenaer Romantik, um das Jahr 1800 herum, was beispielhaft ablesbar erscheint an der Rezeption des Goethe'schen Wilhelm Meister. Dieser Meister endete bekanntlich damit, dass der Held nicht windiger

\footnotetext{
${ }^{2}$ Robert Musil, Gesammelte Werke in neun Bänden, hrsg. v. Adolf Frisé, Reinbek 1981, Bd. I, S. 830.

${ }^{3}$ Siehe dazu detailliert Ortrud Gutjahr, Einführung in den Bildungsroman, Darmstadt 2007, S. 19.

${ }^{4}$ Ortrud Gutjahr, Einführung in den Bildungsroman, a. a. O., S. 27.
} 
Künstler/Schauspieler und erotischer Bohemien, sondern ordentlicher Wundarzt wurde, möglichst mit Festanstellung bei Hofe. Nachdem der Held des Bildungsromans sich, Georg Wilhelm Friedrich Hegel aus seiner Ästhetik zu zitieren, „die Hörner abgestossen“ hatte, sollte er in (feudale oder auch schon bürgerliche, jedenfalls: feste) Bahnen einschwenken. Nur die Jugend erschien jetzt noch poetisch, ihr war deshalb auch die Lyrik zugeordnet, Epos samt Roman stellten dagegen den zum Mann gereiften Protagonisten dar. „Ganz anders verhält es sich dagegen mit dem Roman, der modernen bürgerlichen Epopöe ... Was jedoch fehlt, ist der ursprünglich poetische Weltzustand, aus welchem das eigentliche Epos hervorgeht. Der Roman im modernen Sinne setzt eine bereits zur Prosa geordnete Wirklichkeit voraus ... Eine der gewöhnlichsten und für den Roman passendsten Kollisionen ist deshalb der Konflikt zwischen der Poesie des Herzens und der entgegenstehenden Prosa der Verhältnisse ... ${ }^{5}$ Soweit der nun „objektive“ Berliner Hegel, ehemalige Tutzinger Stiftsgenosse von Schelling und Hölderlin. Der Mann war zum preussischen Staatsphilosophen gereift, ihm war vernünftig, was ist, und nicht mehr das, was sein sollte. Während Novalis' erklärter Vorsatz es rund ein Vierteljahrhundert zuvor noch gewesen war, als er daran ging, den Ofterdingen zu verfassen, einen Roman des ,inneren Weges“ zu schreiben. War der Romantiker doch ,im Juni 1799 ... in spätmittelterlichen Legendensammlungen auf die historisch nicht belegbare Gestalt des Minnesängers Heinrich von Afterdingen gestossen ..., welcher der Sage nach mit Wolfram von Eschenbach, Walter von der Vogelweide und anderen Dichtern am Sängerkrieg auf der Wartburg teilgenommen hatte."6 Novalis' dichterisch-seherisch veranlagter Titelheld tut nun alles, um der festgefügten, bürgerlich-prosaischen Gesellschafts-Bahn seines Philister-Vaters zu entrinnen. Die Goldader eines poetisch glänzenden romantischen Dichterlebens, nicht eine ,poetisierte bürgerliche und häusliche Geschichte“, wie noch bei Goethe, wollte der Bergbauassessor aufgraben. Sein Roman verblieb, wie dann auch der Kafkas, Fragment; erschien, herausgegeben von Novalis' romantischen Freunden, im Jahr 1802. Und dennoch gilt, was erst seit jüngerer Zeit wirklich deutlich geworden ist: „Die durch Schlegel, Tieck und Bülow geschaffene Tradition vom ,fragmentarischen' Denker Novalis ..., ist nicht länger haltbar" - so dezidiert der Herausgeber der Novalis'schen

\footnotetext{
${ }^{5}$ Georg Wilhelm Friedrich Hegel, Ästhetik, 2 Bd., Berlin und Weimar, mit einer Einführung von Georg Lukács, Europäische Verlagsanstalt ohne Jahresangabe, Bd. II, S. 452.

${ }^{6}$ Ortrud Gutjahr, Einführung in den Bildungsroman, a. a. O., S. 92.
} 
Fragmente Richard Samuel im Jahr 1929. Der Paradigmawechsel stützte sich damals auf eine Fülle neuer Materialien. „Inhaltlich bezeugen die Fragmente das breitgefächerte Interessengebiet eines Autors, der sich mit Naturwissenschaften, Philosophie und Geisteswissenschaften, mit praktisch-technischen Problemen (Bergbau) ebenso beschäftigt wie mit erkenntnistheoretischen und ästhetischkünstlerischen Fragestellungen.“ Dieses ,geniale, grossangelegte Modell eines Denkprozesses, an dem sich Weltbild und Denkstruktur der frühromantischen Epoche exemplarisch studieren lassen" "7 , besitzt seine Gültigkeit auch für Novalis bekannten Roman. Dass Carl Schmitt (in Politische Romantik vom Jahr 1919) das Unverbindlich-Symbolische in den Fragmenten glaubte tadeln zu müssen, besitzt seinen Grund auch in der autoritätsgläubigen Urteilsschematik dieses Kronjuristen kommender Nazi-Herrschaft. Auch der Ofterdingen also zeugt von den transdisziplinären Interessen seines Autors. Diese Interessen sind dann bei dem späteren „Kakanier“ Kafka allerdings wiederzufinden, schon, weil der durch seine Begegnung mit Einsteins „Relativitätstheorie“ dem immer schon „drittkulturell“ geprägten, ihm gut bekannten Robert Musil um vieles näher gerückt war, als man bislang realisiert hat. Musil wiederum war nicht nur gut bekannt mit Kafka, sondern auch mit Ernst Weiss. Mit diesem wiederum war Kafka nicht nur als Autor und Zeitgenosse, sondern sogar als Duzfreund verbunden gewesen beide ausserordentlich interessiert an den neuentdeckten Röntgenstrahlen.

\footnotetext{
${ }^{7}$ Richard Samuel zitiert nach Kindlers Lexikon der Weltliteratur, a. a. O., Bd. 12, S. 528.
} 
Open Access Dieses Kapitel wird unter der Creative Commons Namensnennung 4.0 International Lizenz (http://creativecommons.org/licenses/by/4.0/deed.de) veröffentlicht, welche die Nutzung, Vervielfältigung, Bearbeitung, Verbreitung und Wiedergabe in jeglichem Medium und Format erlaubt, sofern Sie den/die ursprünglichen Autor(en) und die Quelle ordnungsgemäß nennen, einen Link zur Creative Commons Lizenz beifügen und angeben, ob Änderungen vorgenommen wurden.

Die in diesem Kapitel enthaltenen Bilder und sonstiges Drittmaterial unterliegen ebenfalls der genannten Creative Commons Lizenz, sofern sich aus der Abbildungslegende nichts anderes ergibt. Sofern das betreffende Material nicht unter der genannten Creative Commons Lizenz steht und die betreffende Handlung nicht nach gesetzlichen Vorschriften erlaubt ist, ist für die oben aufgeführten Weiterverwendungen des Materials die Einwilligung des jeweiligen Rechteinhabers einzuholen.

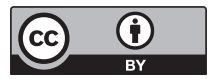

